\title{
Dissection of an aneurysmal ascending aorta in association with coarctation of the aorta
}

\author{
ROBERT A M LAWSON AND ANTHONY FENN \\ From the Cardio-Thoracic Unit, Wythenshawe Hospital, Manchester, UK
}

\begin{abstract}
A previously fit 21-year-old man presented with severe central chest pain. Clinical, electrocardiographic, and echocardiographic examination confirmed a dissection of an aneurysmal ascending aorta in the presence of previously undiagnosed severe aortic coarctation. Initial aortic dissection had occurred five days before admission. The medical and staged surgical management of the case are presented. Surgical survival with such a combination of lesions does not appear to have been previously recorded.
\end{abstract}

In a classic paper on 200 necropsy cases of aortic coarctation collected over 137 years Abbott $(1927 / 8)$ noted that $20 \%$ of the deaths were from rupture of the aorta or, rarely, of the heart itself. In a later series of 104 necropsies similar aortic rupture accounted for $23 \%$ of the deaths (Reifenstein et al, 1947). Isolated cases of ruptured aortic aneurysm in association with aortic coarctation have been reported since then, with successful surgical management of some of those with ruptured mycotic aneurysms distal to the coarctation (Probert, 1956; Cossette et al, 1969; Nikaidoh et al, 1973).

We have been unable to trace a recorded case of successful surgical management of rupture of the aneurysmal ascending aorta with coexisting severe coarctation.

\section{Case report}

A previously fit 21-year-old truck driver developed sudden severe central chest pain as he lifted a $7 \mathrm{~kg}$ carton of margarine. He was admitted to hospital where severe pain persisted for 13 hours despite sedation.

The patient was in good general condition but had a coarctation of the aorta with barely palpable femoral pulses, a well-developed chest wall collateral circulation, and an arm blood pressure of $180 / 100 \mathrm{mmHg}$. A chest radiograph was thought to show some cardiac enlargement and rib notching consistent with aortic coarctation but to be otherwise normal.

Pain recurred intermittently after 72 hours in the central chest and also in the left shoulder and neck. His circulatory state remained stable, although his blood pressure remained at 150-190/ $70-100 \mathrm{mmHg}$. After a further episode of pain he was transferred to a regional cardiothoracic unit five days after the onset of his symptoms.

On admission to the regional unit his general condition remained good despite the pain. His build was normal without any stigmata of Marfan's syndrome. The previous clinical findings were confirmed. An easily palpable thrill was noted over the right carotid artery and a diastolic murmur was heard down the left sternal margin. Air entry to the chest was good and abdominal examination was normal. His haemoglobin was $13.8 \mathrm{~g} / \mathrm{dl}$, white cell count $13.3 \times 10^{9} / 1$, serum electrolytes were normal, and blood urea $11.5 \mathrm{mmol} / 1$. Echocardiography (figs 1 and 2) showed a large pericardial effusion, anterior mitral leaflet diastolic flutter indicating severe aortic regurgitation, a vigorous left ventricle (calculated cardiac output of 14.81 / $\mathrm{min}$ ), and some enlargement of the aortic root (diameter $42 \mathrm{~mm}$ ) but no double aortic wall echo. The ECG showed sinus rhythm, axis of $-30^{\circ}$, left ventricular hypertrophy, and ST changes consistent with pericarditis. The chest radiograph (fig 3) confirmed pronounced cardiomegaly, bilateral rib notching, and considerable widening of the mediastinum.

A clinical diagnosis was made of dissection of the aneurysmal ascending aorta with aortic regurgitation and pericardial effusion in association with coarctation of the aorta. Angiography was not done at this stage as it was considered to be too hazardous. We elected to resect the coarctation as an initial emergency procedure and to investi- 


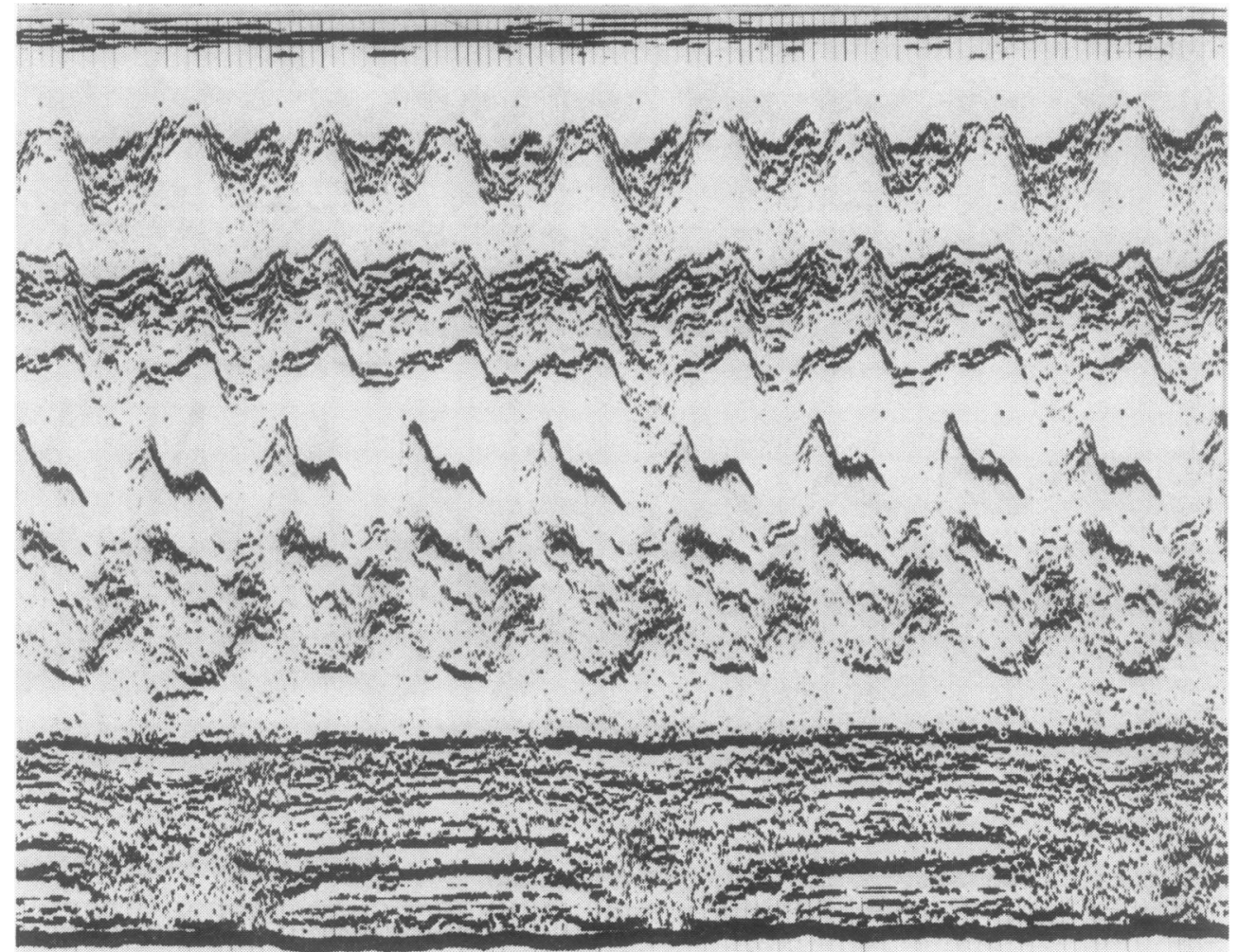

Fig 1 Admission echocardiogram showing a large pericardial fluid collection. Left ventricular action is vigorous.

gate and deal with the ascending aortic dissection at a later date. Immediately after admission the patient's systolic blood pressure was reduced to $100 \mathrm{mmHg}$ with intravenous sodium nitroprusside.

On 30 November 1977 resection of his aortic coarctation was carried out through a left fourth rib thoracotomy. A tense bluish pericardial effusion was confirmed at operation and left severely alone. A dissection of the ascending aorta was seen to extend to, but not beyond, the coarctation that lay in the classical site just distal to the left subclavian artery. After resection of the coarctation, aortic continuity was established with a $4 \mathrm{~cm}$ long $14 \mathrm{~mm}$ diameter woven Dacron graft. Artificial ventilation and sodium nitroprusside treatment were discontinued 12 hours after operation, arm blood pressure thereafter being maintained at 110-120/60-70 $\mathrm{mmHg}$ with propranolol and methyldopa.

Over the next four days the patient had no more central chest pain, his general condition remained stable, and the murmur of the aortic regurgitation remained unchanged. The chest radiograph, however, showed minimal further mediastinal widening

A left heart study on 5 December 1977 showed a left ventricular pressure of $130 / 20 \mathrm{mmHg}$ and an ascending aortic pressure of $120 / 65 \mathrm{mmHg}$. An aortic root injection of contrast medium confirmed gross dilatation of the ascending aorta which narrowed inferiorly to a slightly enlarged aortic ring. Superiorly the aorta became relatively normal in size below the innominate artery origin. Moderate to severe aortic regurgitation into a very dilated left ventricle was shown. No definite dissection could be seen.

Immediately after this investigation an exploration of the ascending aorta and aortic valve was begun. After cannulation of the right femoral artery for arterial return, the pericardium was opened to release $400 \mathrm{ml}$ of old blood and fibrin. The ascending aorta measured $8 \mathrm{~cm}$ in diameter and narrowed superiorly and inferiorly. A posterior dissection originating $1.5 \mathrm{~cm}$ above the left 

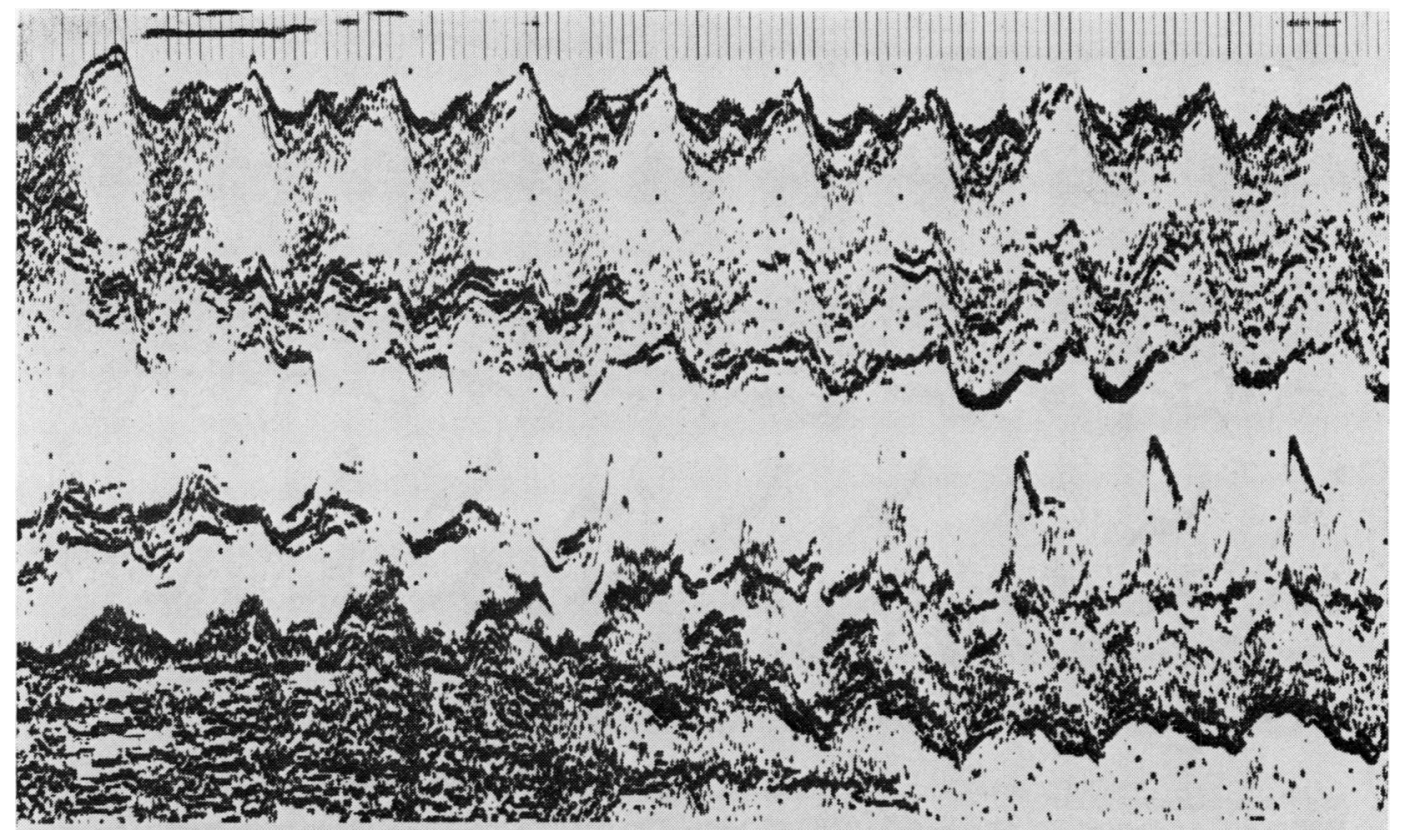

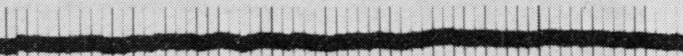

Fig $2 A$ wide aortic root is shown but there is no clear evidence of dissection. Fine diastolic oscillation of anterior mitral valve leaflet indicates aortic regurgitation.

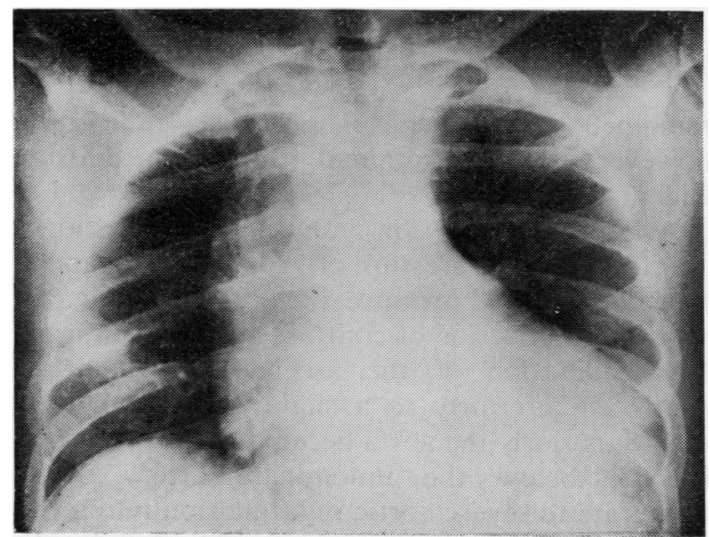

Fig 3 Chest radiograph on admission to regional cardiothoracic unit. A considerable increase in mediastinal and cardiac size is noted.

coronary orifice had tracked down to, but not around it. The dissection had extended leftwards around the aorta, but no definite exit point into the pericardium could be seen anteriorly. Superiorly the dissection affected three-quarters of the aortic circumference and had extended up the innominate artery and beyond it around the arch. The aortic valve was tricuspid and the cusps were healthy, but regurgitation resulted from anterior dislocation of the commissure between the nonand left-coronary cusps by the inferior dissection. The left ventricle was moderately hypertrophied and considerably dilated.

With the aid of cardiopulmonary bypass, whole body cooling to $28^{\circ} \mathrm{C}$, and cold hyperkalaemic cardioplegia the ascending aorta was replaced with a $25 \mathrm{~mm}$ preclotted woven Dacron graft after resuspending the dislocated aortic commissure using 4/0 Prolene sutures tied over Teflon buttresses outside the aortic wall. The proximal end of the graft was sutured to the aorta $0.5 \mathrm{~cm}$ above the coronary orifices.

The cardiopulmonary bypass was terminated without difficulty. Continued suture line bleeding ultimately responded to infusion of fresh blood and Teflon wrapping of both suture lines.

After the operation the circulation was supported by minimal doses of dopamine for 24 hours after which artificial ventilation was discontinued. The patient thereafter made an uneventful re- 
covery and was discharged home 16 days after his second operation. He was taking digoxin, $0.25 \mathrm{mg}$, and propranolol, $30 \mathrm{mg}$, daily.

On discharge he had clinical signs of moderate aortic regurgitation with a $2 / 6$ diastolic murmur down the left sternal edge and a blood pressure of $130 / 60 \mathrm{mmHg}$. Echocardiography (fig 4) showed a wide aortic root (43 mm diameter) with eccentric aortic cusp closure, slight left ventricular dilatation with excellent action, and moderately increased cardiac output at $9.9 \mathrm{l} / \mathrm{min}$. The chest radiograph showed a striking reduction in his heart size (fig 5).

Three months after operation he resumed fulltime work and when seen one year later he was asymptomatic with a blood pressure of $140 / 75$ $\mathrm{mmHg}$ in both arms. His chest radiograph showed a further reduction in heart size and his ECG showed improvement on the previously noted signs of left ventricular strain. His diastolic murmur, however, remained unchanged and a supra-aortic contrast injection showed moderate-to-severe aortic regurgitation (fig 6).

Histology of the coarctation specimen showed considerable thickening of both media and intima with some atheroma. The lumen was reduced to a $2 \mathrm{~mm}$ slit at the centre of the specimen. Histology of the ascending aortic aneurysm showed intimal thickening and extensive haemorrhage into the

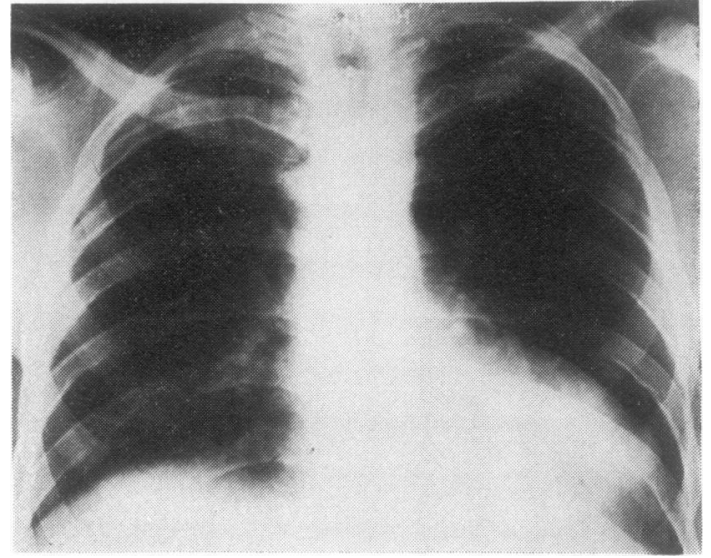

Fig 5 Chest radiograph on discharge showing a pronounced reduction in mediastinal shadowing and some reduction in the cardiac shadow.

media. Where the media was present there was necrosis with loss of nuclear pattern and hyalinisation of the cytoplasm. The adventitia was infiltrated with inflammatory cells, mainly polymorphs but with some plasma cells and leucocytes. The appearances were compatible with dissecting aneurysm of the aorta associated with medial necrosis.

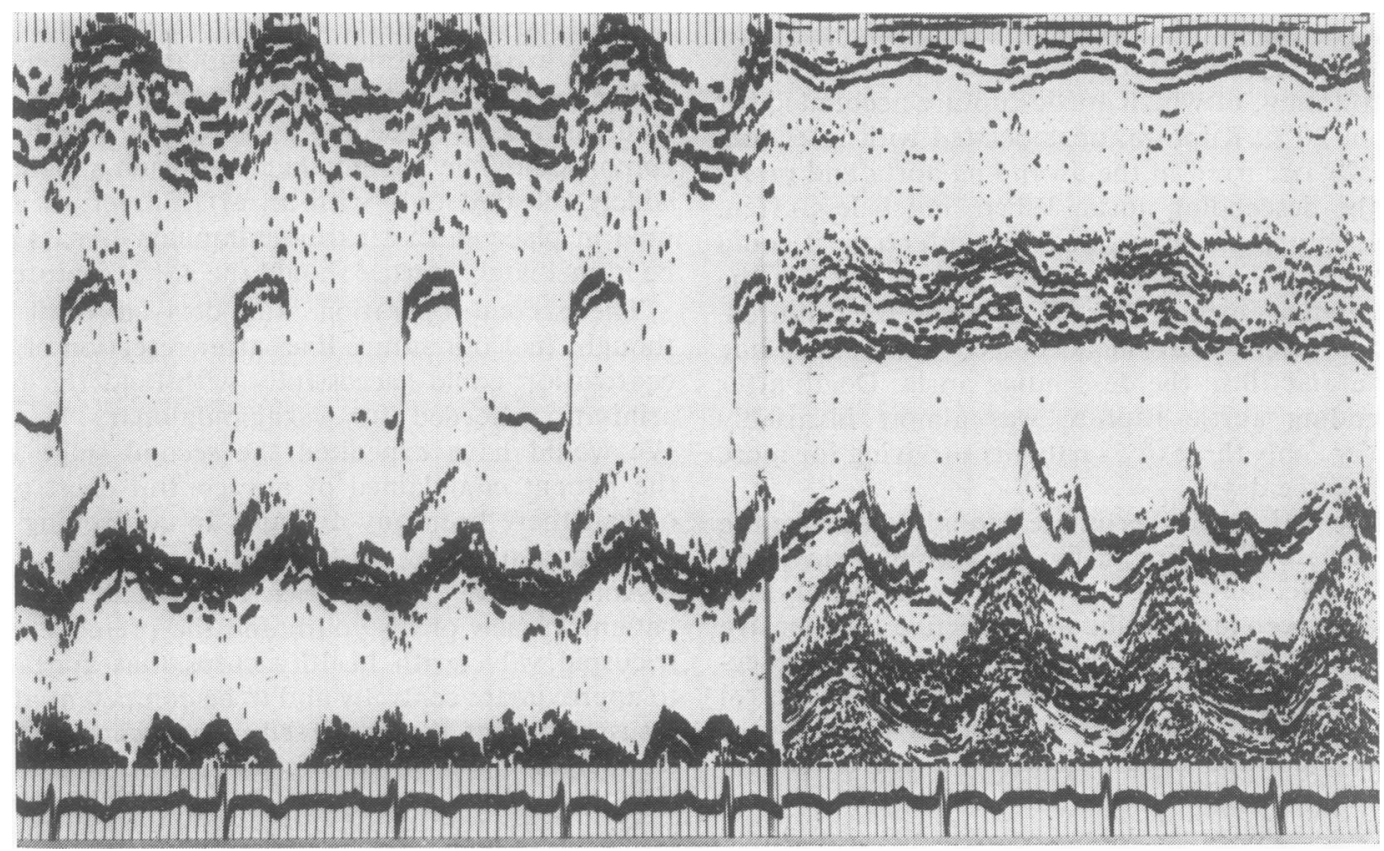

Fig 4 Echocardiogram on discharge shows a wide aortic root with eccentric aortic valve closure. The left ventricle is vigorous and fine mitral oscillations are again shown. 


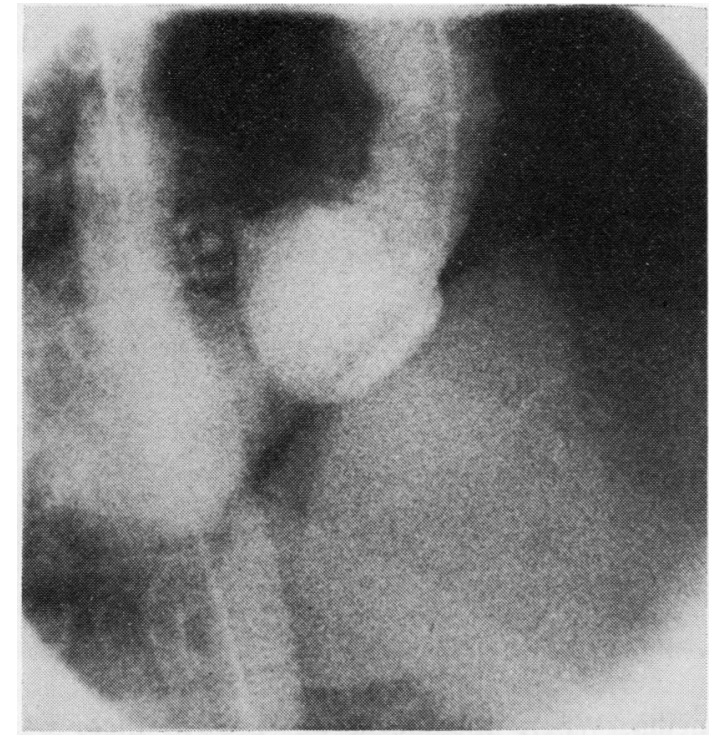

Fig 6 Supra-aortic contrast medium injection one year after operation showing moderate-to-severe aortic regurgitation.

\section{Discussion}

The natural history of untreated aortic coarctation has been well reviewed by Abbott (1928/29) and Reifenstein et al (1947). Of the deaths in these series, $75 \%$ were from complications of the aortic coarctation, of which aortic rupture accounted for about $20 \%$. Rupture, unassociated with infection, usually occurred in the ascending aorta and rarely in the descending aorta. When, much less often, the rupture was associated with infection, it could occur in either the ascending or more often in the descending aorta. Thus 52 of their 62 ruptured, non-infected aneurysms occurred in the ascending aorta and 10 in the descending aorta. Death after ascending aortic rupture was almost invariably sudden, only three of 52 patients surviving for more than three days.

Our patient was fortunate enough to survive the immediate dissection, a five-day hospital stay, and a 10-mile ambulance trip to the regional unit before receiving specific and effective hypotensive treatment. He then survived an emergency resection of his coarctation under hypotensive control and a further four days in hospital under hypotensive control until resection of his ascending aorta was performed.

Management of dissection of the ascending aorta in the presence of severe aortic coarctation poses problems for both the investigating cardi- ologist and the surgeon. Dissection of the ascending aorta in these cases classically originates posteriorly (Abbott, 1928/9), extends as far as the coarctation (Edwards, 1973), and may affect head and arm vessels, as occurred in our case. Extremely weak and delayed femoral pulses and gross chest wall collaterals confirmed a severe aortic coarctation and contraindicated any catheter study or arterial cannulation from the groin. Echocardiography confirmed a large pericardial effusion, aortic root dilatation, and anterior mitral cusp oscillation secondary to aortic regurgitation but did not show a definite dissection. We considered that the clinical, radiological, electrocardiographic, and echocardiographic findings in our patient were sufficiently definite to make a firm diagnosis of dissection of the ascending aorta in the presence of severe aortic coarctation and that angiographic studies at this stage might prove more hazardous than helpful.

Pharmacological (Wheat et al, 1965) and anatomical reduction in after-load by resection of the aortic coarctation seemed the logical initial management. We accepted the considerable risks of delay in resection of the dissected ascending aorta (Daily et al, 1970; Dalen et al, 1974; Strong et al, 1974). Controlled hypotension during coarctation resection was mandatory to avoid damage by aortic clamps to the upper aorta surrounded by mediastinal haematoma. From previous experience we had learnt to resist the temptation to release a haemorrhagic pericardial effusion as this can result in massive bleeding as the aortic tear is decompressed. The haemorrhagic effusion should be widely released only with an arterial return cannula in place so that cardiopulmonary bypass can be immediately started should the aorta rupture.

The second operation was delayed until we thought that our suture lines after resection of the coarctation could successfully withstand the heparinisation needed for cardiopulmonary bypass. We would have expedited the second stage had the patient complained of any central chest pain or had there been any difficulty in controlling his blood pressure.

Aortic valve repair was performed on our patient in view of his youth and the presence of a tricuspid valve with healthy cusps that appeared to approximate centrally and to be competent after resuspension of the prolapsed commissure. Teflon cloth insertion into the inferior dissection to provide a fixed annulus was not used (Collins and Cohn, 1973). Our patient's moderate-to-severe postoperative aortic regurgitation may be related to some dilatation of the annulus or possible dis- 
tortion, or both, caused by the external Teflon wraps used to control suture line bleeding.

Medial necrosis in the ascending aorta adjacent to areas of the aortic rupture was noted in 12 of the 13 patients in Abbott's series on whom histological examinations were performed. Interruption and diminution of the elastica with connective tissue increase and hyaline and fatty degeneration were specifically noted. The frequent finding of a dilated ascending aorta, especially thin walled at its root and associated with a bicuspid aortic valve in $50 \%$ of her cases suggested to Abbott a "primary deficiency in the vascular anlage." Medial necrosis with hyaline degeneration, basophilic appearance, or cystic change was also noted by Reifenstein and co-workers (1947).

An association between cystic medial necrosis and bicuspid aortic valve has also been noted (McKusick et al, 1957) and thought to be similar to that between coarctation and bicuspid aortic valve (McKusick, 1972a). Although the medial necrosis was at first thought to be the result of a non-specific aortic response to haemodynamic stress (McKusick et al, 1957), similar histological findings in arteries under less haemodynamic stress -for example, the pulmonary artery, and in the ascending aorta of a father and son who both had bicuspid aortic valves and died after rupture of their ascending aortic aneurysm-have subsequently caused McKusick (1972b) to suggest that the aetiology may be congenital.

Gross aneurysms of the sinuses of Valsalva have been recorded as developing within two years of replacement of the aortic valve and ascending aorta in a teenager with Marfan's syndrome (Symbas et al, 1971). The lateralised blood flow through an aortic Starr-Edwards ball valve prosthesis impinging on the cuff of aorta left between the aortic annulus and the prosthetic graft was thought in that case to have contributed to aneurysm formation. Our patient had no outward manifestations of Marfan's syndrome and retains his own central orifice flow valve. His aortic histology suggests that he may be at some risk in the future from development of an aneurysm of the aortic root but he should be protected from this complication by the proximal Teflon wrap inserted at operation. Although at present haemodynamically well compensated, the residual aortic regurgitation will certainly necessitate aortic valve replacement in the future.

We thank Drs Bray, Croxson, and Beton and Mr J F Dark for their advice and help in the management of this case.

\section{References}

Abbott, M E (1927/8). Coarctation of the aorta of the adult type. American Heart Journal, 3, 574-618.

Collins, J J, and Cohn, L H (1973). Reconstruction of the aortic valve. Archives of Surgery, 106, 35-37.

Cossette, R, Davignon, A, and Stanley, P (1969). Rupture of the aortic aneurysm in a three-and-a-halfyear-old-child with coarctation of the aorta. Canadian Medical Association Journal, 100, 257-261.

Daily, P O, Trueblood, H W, Stinson, E B, Wuerflein, $R$ D, and Shumway, N E (1970). Management of acute aortic dissection. Annals of Thoracic Surgery, 10, 237-247.

Dalen, J E, Alpert, J S, Cohn, L H, Black, H, and Collins, J J (1974). Dissection of the thoracic aorta. Medical or surgical therapy? American Journal of Cardiology, 34, 803-808.

Edwards, J E (1973). Aneurysms of the thoracic aorta complicating coarctation. Circulation, 48, 195-201.

McKusick, V A, Logue, R B, and Bahnson, H T (1957). Association of aortic valvular disease and cystic medial necrosis of the ascending aorta. Circulation, 16, 188-194.

McKusick, V A (1972a). Heritable Disorders of Connective Tissue. 4th edn. C V Mosby, St Louis.

McKusick, V A (1972b). Association of congenital bicuspid aortic valve and Erdheims cystic medical necrosis. Lancet, 1, 1026-1027.

Nikaidoh, H, Idriss, F S, and Riker, W L (1973). Aortic rupture in children as a complication of coarctation of the aorta. Archives of Surgery, 107, 838-841.

Probert, W R (1956). Coarctation of the aorta associated with an aneurysm leading into the bronchial tree, treated by aortic resection and grafting. Proceedings of the Royal Society of Medicine, 49, 729-731.

Reifenstein, G A, Levine, S A, and Gross, R E (1947). Coarctation of the aorta. American Heart Journal, 33, 146-168.

Strong, W W, Moggio, R A, and Stansel, H G (1974). Acute aortic dissection. Twelve year medical and surgical experience. Journal of Thoracic and CardioVascular Surgery, 68, 816-821.

Symbas, P N, Raizner, A E, Tyras, D H, Hatcher, C R, Inglesby T V, and Baldwin, B J (1971). Aneurysms of all sinuses of Valsalva in patients with Marfan's syndrome. Annals of Surgery, 174, 902907.

Wheat, N W, Palmer, R F, Bartley, T D, and Seelman, R C (1965). Treatment of dissecting aneurysms of the aorta without surgery. Journal of Thoracic and Cardio-Vascular Surgery, 50, 364-373.

Requests for reprints to: Robert A M Lawson, FRCS, Cardio-Thoracic Unit, Wythenshawe Hospital, Manchester. 